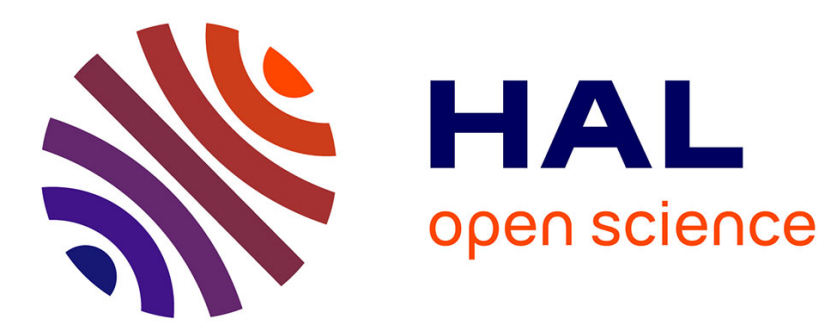

\title{
Locomotion Approach of REMORA: a REonfigurable MObile Robot for Manufacturing Applications
}

\author{
Hai Yang, Sébastien Krut, François Pierrot, Cédric Baradat
}

\section{To cite this version:}

Hai Yang, Sébastien Krut, François Pierrot, Cédric Baradat. Locomotion Approach of REMORA: a REonfigurable MObile Robot for Manufacturing Applications. IROS: Intelligent RObots and Systems, Sep 2011, San Francisco, CA, United States. pp.5067-5072, 10.1109/IROS.2011.6094897 . lirmm00641618

\section{HAL Id: lirmm-00641618 \\ https://hal-lirmm.ccsd.cnrs.fr/lirmm-00641618}

Submitted on 16 Nov 2011

HAL is a multi-disciplinary open access archive for the deposit and dissemination of scientific research documents, whether they are published or not. The documents may come from teaching and research institutions in France or abroad, or from public or private research centers.
L'archive ouverte pluridisciplinaire HAL, est destinée au dépôt et à la diffusion de documents scientifiques de niveau recherche, publiés ou non, émanant des établissements d'enseignement et de recherche français ou étrangers, des laboratoires publics ou privés. 


\title{
Locomotion Approach of REMORA: a REonfigurable MObile Robot for manufacturing Applications
}

\author{
Hai Yang* , Sébastien Krut ${ }^{\dagger}$, Cédric Baradat* and François Pierrot ${ }^{\dagger}$ \\ * Industrial Systems Unit, Tecnalia \\ 34960, Montpellier France \\ Email: (hai.yang, cedric.baradat)@tecnalia.com \\ $\dagger$ Univ. Montpellier 2, CNRS, LIRMM, 161 rue Ada \\ 34392 Montpellier, France \\ Email: (krut, pierrot)@lirmm.fr
}

\begin{abstract}
This paper presents the locomotion approach of a novel quadruped robot which is able to carry various effectors for achieving manufacturing tasks in large workspaces. Equipped with lockers on some of the passive joints and clamping devices at the end of its limbs, this quadruped uses eight actuators for achieving manufacturing tasks as well as locomotion tasks. In the following sections, we first present the proposed robot and its two working modes. Then, the locking strategy of the robot is formulated as an optimization problem. Also, a practical method for managing the limbs swinging movement is addressed. At last, the presented approach is applied on two concrete examples. Possessing a low degree of kinematic redundancy, the proposed quadruped shows a reasonable locomotion capacity which allows it to achieve locomotion with respect to some extra constrains in its workspaces.
\end{abstract}

\section{INTRODUCTION}

Modern industry requires manufacturing systems to be more reconfigurable, flexible and agile to adapt to the increasing competitive climate with sophisticated customers demands [1]. In industrial fields such as automobile assembly lines or semiconductor manufacturing processes, automated robotic systems have been widely integrated. But in the sections such as aeronautics and shipbuilding, train manufacturing where large workspaces are required, the traditional stationary-base robots cannot be used.

Therefore, the concept of mobile manipulator, classical serial robot arms mounted on a mobile base, has been considered for the automation of applications like welding, inspection, painting etc [2], [3]. However, most of these solutions will fail when a high precision and/or stiffness are required for applications like drilling or milling operations.

Meanwhile, parallel kinematic machines (PKMs) which have great potential to provide high rigidity and motion dynamics suffer from their inherent limited operational workspace. The combination of PKMs and various mobile bases have been proposed in order to apply PKMs to the industry where a large operational workspace is required. The mobility of the base could be provided by linear guide ways [4], [5], wheeled mobile robots [6] or tracked mobile robots. On the one hand, long linear guide ways with high precision and stiffness represent high costs and tedious infrastructure adjustments of workshops. On the other hand, beside the need of independent control for the mobile base, wheeled and tracked mobile robots, possessing lower degrees of freedom (DOF), have limited obstacle crossover ability.

Legged robots have attracted attention because of their relatively good terrain pass-over capacity [7]. However, few of them have been used to solve industrial problems due to the complexity of the control and the lack of reliability: the conventional legged robot requires lots of degrees of freedom. In the literatures, several attempts were carried out to use the mobility of PKMs for locomotion purposes [8], [9]. Such approach helps to build legged robots with reduced degree of freedom, however most of these research lines are away from the manufacturing purposes.

Designing a legged robot for manufacturing purpose is very different from designing a legged robot for exploration of hazardous unknown environments. Several techniques and the topological analysis for designing industry oriented legged robots are addressed in [10]. The proposed quadruped which combines the mobility of legged robots and advantages of PKMs is able to carry various effectors for achieving manufacturing tasks in large workspaces. Equipped with lockers on some of the passive joints and clamping devices at the end of its limbs, this quadruped uses eight actuators for achieving manufacturing tasks as well as locomotion tasks.

In the following sections, we first introduce the whole system of the REconfigurable MObile Robot for manufacturing Applications (REMORA) and its working modes. Then the locomotion problem of the robot is managed by dealing with the issues of the locking strategy and the limb swinging movement. At last, the presented locomotion approach is applied to two examples where certain constrains in the workspaces are added.

\section{DESCRIPTION OF THE SYSTEM}

\section{A. Overall Description of REMORA}

The whole system consists of the robot itself and the clamping pins fixed on the supporting media which can be the floor, the fixture of workpieces or other structures in the workshop (Fig. 1). The CAD Model of REMORA is shown in Fig. 2. Lockers are equipped on some of the passive joints of REMORA. Furthermore, there is a clamping device on the extremity of each limb, which provides moderate clambering capacity to the robot. 


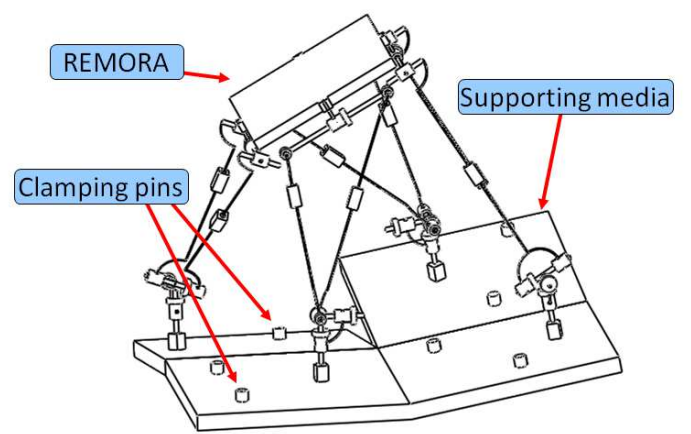

Fig. 1: General figure of the whole system

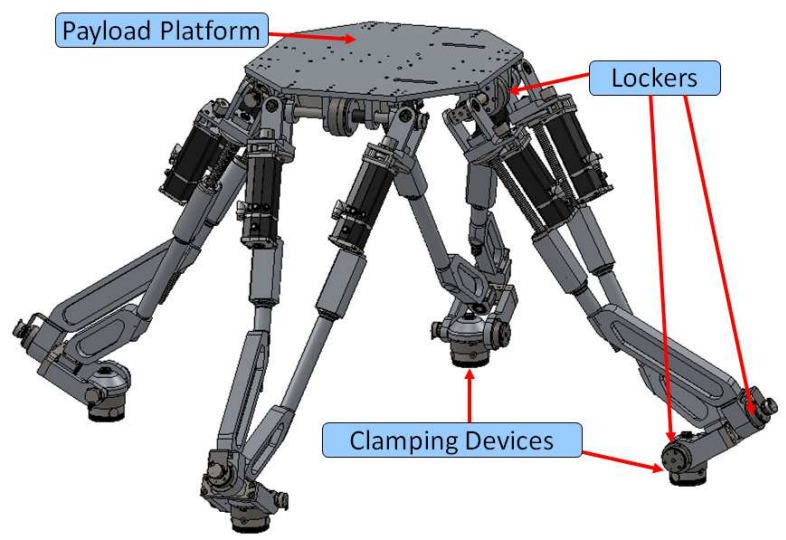

Fig. 2: CAD Model of REMORA

\section{B. Geometrical Description of the Robot}

The geometry of REMORA can be described by the parameters in Fig. 3. Four branches are symmetrically mounted on the Payload Platform (PP), the axes of the last joints of every branch being coplanar. The branch $i$ is connected to the $\mathrm{PP}$ at $P_{L i} P_{R i} . P_{C i}$ is the middle point of $P_{L i} P_{R i} . r_{p}$ is the radius of the circle which passes through the connecting points $P_{L i}, P_{R i}$ of all branches. $d_{p}$ denotes the distance between the opposite limbs. $d$ is the distance between $P_{L i}$ and $P_{R i}$.

Fixed at the center of the circle $P_{O}$, the frame $\mathscr{R}_{P}\left(P_{O}, u_{p}, v_{p}, w_{p}\right)$ is attached on the PP with the u-axis pointing to $P_{c_{I}}$. The frame $\mathscr{R}_{B}\left(B_{O}, u_{b}, v_{b}, w_{b}\right)$ is the world frame which is fixed on the supporting media. Limbs frame $\mathscr{R}_{A_{i}}\left(A_{i}, u_{a_{i}} v_{a_{i}} w_{a_{i}}\right)_{(i=1,2 \ldots 4)}$ is defined for each limbs, with its origin located at the point $A_{i}$ of the $i_{t h}$ limb.

Variable $x\left(x_{P} ; y_{P} ; z_{P} ; \alpha_{P} ; \beta_{P} ; \gamma_{P}\right)$ links to the pose of the PP. It describes the pose of the frame $\mathscr{R}_{P}$ with respect to the world frame $\mathscr{R}_{B}$. $\left[x_{p}, y_{p}, z_{p}\right]^{T}$ is the position vector of the point $P_{O}$ with respect to $\mathscr{R}_{B} . \alpha_{P}, \beta_{P}$ and $\gamma_{P}$ are the rotations about the fixed $u_{b}, v_{b}$ and $w_{b}$ axes of $\mathscr{R}_{B}$. In the same way, $x_{a_{i}}\left(x_{A_{i}} ; y_{A_{i}} ; z_{A_{i}} ; \alpha_{A_{i}} ; \beta_{A_{i}} ; \gamma_{A_{i}}\right)_{(i=1,2 \ldots 4)}$ links to the pose of branch extremity (BE) of the $i_{\text {th }}$ branch.

The revolute joints variable $s=[q 6, q 5 R, q 5 L, q 3 R, q 3 L$, $q 2, q 1]^{T}$ corresponds the joints values in one limb. The subscript $L_{i(i=1,2 \ldots 4)}$ is added for referencing a specific limb.

The actuator variable $q=\left[q L_{L_{1}}, q R_{L_{1}}, q L_{L_{2}}, q R_{L_{2}}, q L_{L_{3}}\right.$, $\left.q R_{L_{3}}, q L_{L_{4}}, q R_{L_{4}}\right]^{T}$ represents the generalized actuation

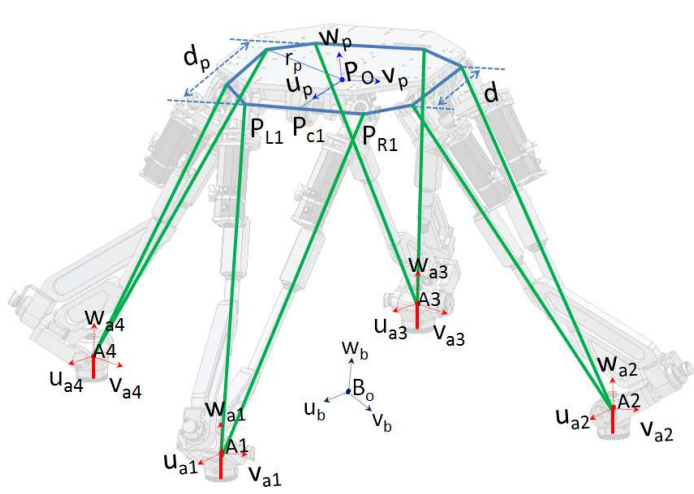

(a) General coordinates of robot

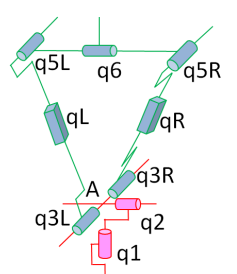

(b) Joints variables

Fig. 3: Configuration and notations of REMORA

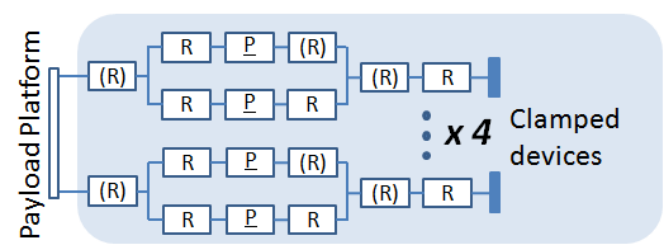

(a) PP mode

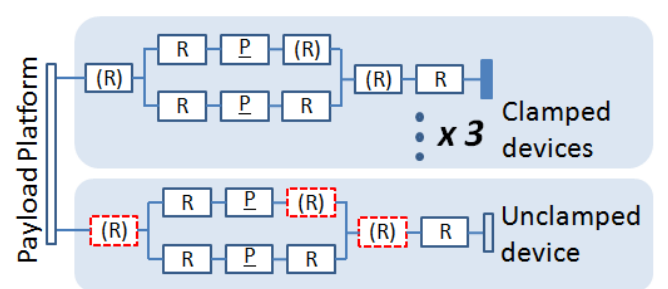

(b) BE mode

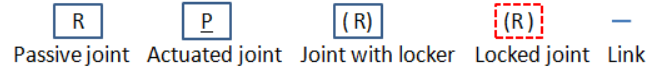

Fig. 4: Joint-and-Loop graph of two working modes

vector which corresponds to the displacement of the eight prismatic joints in the four limbs of the robot.

\section{Working Mode}

The working modes of REMORA can be roughly distinguished as: Payload Platform (PP) mode and Branch Extremity (BE) mode.

PP Mode: As illustrated in Fig. 4 (a), all branches of the robot are attached to the supporting media. Each branch possesses six DOFs; two of the them are actuated. The PP of the robot, which has six DOFs, is actuated by eight actuators located in the four branches connected to the 
base. Therefore, the robot can be considered as a six DOFs PKM with actuation redundancy in this mode. Thanks to the clamping device located at each branch extremity, the workspace of the robot are not limited by the robot's center of gravity neither the friction between the foot and the ground.

BE Mode: One branch of the robot is detached from the base in order to reach another supporting pin. The other three branches remain attached to the base. The PP, with six DOFs, are still fully controllable by actuators located in the three attached branches. Meanwhile the passive DOFs in the swing branch should be eliminated in order to control its BE link. To do so, the corresponding lockers will be activated. Then REMORA can be considered as a hybrid robot which consists a six DOFs PKM and an extra two DOFs arms amounted on the PP. There are eight actuators, both the ones in the supporting branches and in the swinging branch, contribute to position the BE of the swinging leg in 3-D space with given orientation. Thus, REMORA can be called kinematic redundant in the BE mode.

\section{STRATEGY FOR LOCOMOTION}

Fig. 5 (a) shows a working scenario which presents one operation cycle from one work location to another. The locomotion of REMORA is achieved by relocating the limbs one after another. For each step, the supporting pattern of the robot is different. Fig. 5 (b) shows the variation of the supporting pattern in one step. The quadrilateral forms a supporting pattern. Blue cercles represent the clamping pins among which the solid dots represent the clamped pins. As only one limb will be in swinging at once, there should only be one supporting point changed between supporting pattern $\alpha$ and $\beta$.

The swinging limb will be detached from the supporting pin, moved to the next supporting pin, and then attached to it. During all these stages, the passive DOFs in this limb are locked, which means that each locked joint remains the same value during the limb swinging phase. In this section, we will first explain how the locking values of the lockable joints are determined. Then the approach that has been used

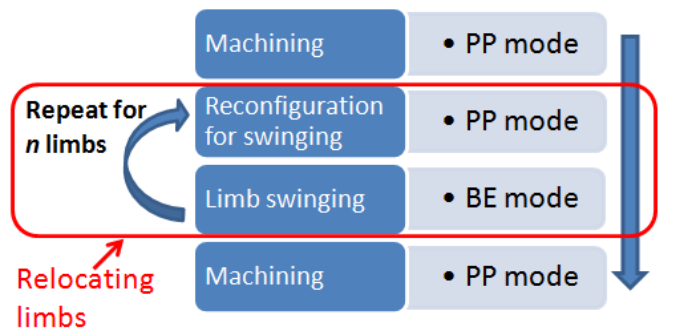

(a) General scenario

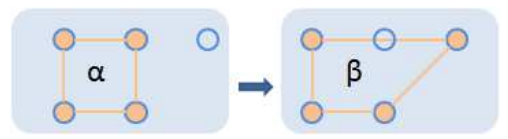

(b) Supporting pattern variation in one step

Fig. 5: General scenario and typical step of REMORA for achieving the limb swinging movement will be addressed as well.

\section{A. Locking Strategy}

1) Nonlinear Equality Constrains: The reconfiguration phase in Fig. 5(a) is for preparing the detachment of the swinging branch. During this phase, all limbs of the robot are still attached on the supporting pins. Before locking the corresponding lockable joints, the PP is supposed to move to a specific pose in order to have the concerned lockable joints equaling desired locking values. The concerned lockable joints are denoted by $b=[q 6, q 3 L, q 2]^{T}$ which is a subset of the variable $s$. Notice that there is no locker amounted on revolute joint $q 1$, because the value of $q 1$ will not influence the clamping process. The relation between variable $b_{i}$ and $x$ can be expressed as:

$$
f_{1}\left(b_{i}, x, x_{a_{1}}, x_{a_{2}}, x_{a_{3}}, x_{a_{4}}\right)=0
$$

where $f_{l}$ is an implicit function, $i$ is the index of the swinging limb and $x_{a_{j}(j=1,2 \ldots 4)}$ denotes the poses of all four branch extremities which is mentioned as a 'supporting pattern'.

Equation (1) shows that the relation between $x$ and $b$ also depends on the values of $x_{a_{j}(j=1,2 \ldots 4)}$. With all the limbs attached to the ground, $x_{a_{j}(j=1,2 \ldots 4)}$ can be considered as constants. Given the value of $x, b_{i}$ is calculated by the inverse kinematics models:

$$
b_{i}=B_{i} \operatorname{IKMX}(x)
$$

where $B_{i} I K M X$ is one of the explicit forms of (1)

The values of $b_{i}$ will not change during the limbs swinging phase, which means the poses of the PP should be chosen in the way that the following constrain is satisfied:

$$
B_{i} I K M X^{\alpha}\left(x_{1}\right)-B_{i} I K M X^{\beta}\left(x_{2}\right)=0
$$

where $x_{1}$ and $x_{2}$ are the key poses of the PP for detachment and attachment respectively. $B_{i} I K M X^{\mu}$ is for referencing the inverse model which corresponds to the supporting pattern $\mu$. In this section, the problem is presented based on the step from the supporting pattern $\alpha$ to the supporting pattern $\beta$.

2) Nonlinear Inequality Constrains: With all limbs attached to the supporting pattern $\alpha$, the robot works as a PKM. $x_{1}$, the detaching poses of the PP, should be in the workspace of such PKM. Also after swinging the limb, the $\mathrm{BE}$ of the swinging limb is ready to be attached to the clamping pin. $x_{2}$, the attaching poses of the PP, should be in the workspace of the PKM which is based on the supporting pattern $\beta$.

The workspace of the machine is determined by the limiting lengths of all the actuators and the limiting angular travel limits of all the revolute joints. To formulate these constraints sets determining the workspaces, the inverse kinematic models of the robot are derived. For a given pose of the PP, the length of the actuators $q$ is calculated by:

$$
q=Q I K M X(x)
$$

where $Q I K M X$ is the inverse kinematics models based on the vectors loop equations [11]. 


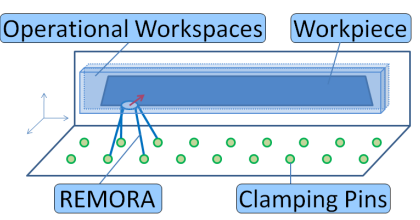

(a) Sideward workspaces

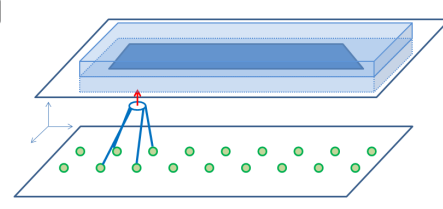

(b) Upward workspaces
Fig. 6: Workshop settings

And, the values of all the revolute joints can be obtained by the inverse kinematics models:

$$
s_{i}=\operatorname{SIKMX}(x)
$$

where SIKMX is formulated by the vector projection approach $^{1}$.

Then the reachable workspace of the robot is defined by the following constrains:

$$
\begin{aligned}
& q_{\text {min }}<Q I K M X(x)<q_{\max } \\
& s_{\text {min }}<\operatorname{SIKMX}(x)<s_{\text {max }}
\end{aligned}
$$

where $q_{\min }$ and $q_{\max }$ are the vectors which contain the limits of all the actuators, $s_{\min }$ and $s_{\max }$ are the vectors which contain the angular travel limits of all the revolute joints.

For different applications, the location of workspaces of REMORA can be different according to the way that the tools are installed on the PP. Fig. 6 shows two kinds of workshop settings. The blue zone can be considered as the operational workspaces where the tool of the robot is in contact with the workpiece during operations. In order to avoid collisions with the workpiece, the robot should not enter into such zone during the locomotion phase. For respecting this kind of requirements, the constrains can be taken into account by adding limits directly on the PP pose variable $x$ :

$$
x_{\min }<x<x_{\max }
$$

where $x_{\min }$ and $x_{\max }$ are vectors with six elements which correspond the limiting values of the position and orientation parameters of the PP.

3) Determining Proper key Poses for the PP: Theoretically, any two poses of the PP, which are in the reachable workspace and satisfy (3), can be chosen as detaching and attaching poses.

For practical reasons, we try to minimize the distance between the detaching pose and the attaching pose in the actuation space. Furthermore the poses which make the actuators close by their median positions are preferred.

The vector which points to the median position of the actuation space is defined as:

$$
\hat{q}=\frac{q_{\min }+q_{\max }}{2}
$$

\footnotetext{
${ }^{1}$ The formulation of the inverse kinematics models: $B_{i} I K M X, S I K M X$
} and $Q I K M X$ is presented in [10].
In the actuation space, the distance between the vectors of the detaching position and the median position can be expressed as:

$$
d_{1}=\left\|Q I K M X^{\alpha}\left(x_{1}\right)-\hat{q}\right\|
$$

And for the attaching position, such distance is as follows:

$$
d_{2}=\left\|Q I K M X^{\beta}\left(x_{2}\right)-\hat{q}\right\|
$$

Similarly, the distance between the detaching and the attaching position in actuation space is as follows:

$$
d_{3}=\left\|Q I K M X^{\alpha}\left(x_{1}\right)-Q I K M X^{\beta}\left(x_{2}\right)\right\|
$$

Combining the previous models, the objective function $f$ can be written as follows:

$$
f\left(x_{1}, x_{2}\right)=k_{1} d_{1}+k_{2} d_{2}+k_{3} d_{3}
$$

where $k_{1}, k_{2}$ and $k_{3}$ are the weight coefficients.

Now the locking issue can be written as finding the minimum of the problem specified by:

$$
\min _{\left(x_{1}, x_{2}\right)} f\left(x_{1}, x_{2}\right)
$$

such that:

$$
B_{i} I K M X^{\alpha}\left(x_{1}\right)-B_{i} I K M X^{\beta}\left(x_{2}\right)=0
$$

and

$$
\begin{gathered}
x_{1 \text { min }}<x_{1}<x_{1 \text { max }} \\
x_{2 \text { min }}<x_{2}<x_{2 \max } \\
q_{\text {min }}<Q I K M X^{\alpha}\left(x_{1}\right)<q_{\text {max }} \\
q_{\text {min }}<Q I K M X^{\beta}\left(x_{2}\right)<q_{\text {max }} \\
s_{\text {min }}<\operatorname{SIKMX}^{\alpha}\left(x_{1}\right)<s_{\text {max }} \\
s_{\text {min }}<\operatorname{SIKMX}^{\beta}\left(x_{2}\right)<s_{\text {max }}
\end{gathered}
$$

\section{B. Limb Swinging Movement}

During the limb swinging phase, REMORA works in BE mode. It can be considered as a hybrid robot which consists of a 2-DOFs arms amounted on a 6-DOFs PKM.

By minimizing the objective function $f\left(x_{1}, x_{2}\right)$ in (13), the corresponding detaching pose $\left(x_{1}\right)$ and its attaching pose $\left(x_{2}\right)$ can be determined.

From $x_{1}$ to $x_{2}$, the movement of the PP is generated by the six actuators located in the three attached limbs. Thanks to the relation (4), the actuators values $q^{\alpha}$ and $q^{\beta}$ which correspond to $x_{1}$ and $x_{2}$ can be determined accordingly with supporting pattern $\alpha$ and $\beta$.

Furthermore, (2) is used for calculating the locking values of the concerned lockable joints $\left(b_{i}\right)$. Since that $b_{i}$ remains constant during the limb swinging phase and $x$ is a pose in the given trajectory of the PP, the pose of the swinging $\mathrm{BE}$ link is determined by (1).

During the limb swinging phase, the six DOFs of the swinging BE link are controlled by eight actuators, which means the robot is kinematically redundant. If the trajectory following of this BE link is expected in the whole workspace, the inverse kinematic model which considers the concerned 


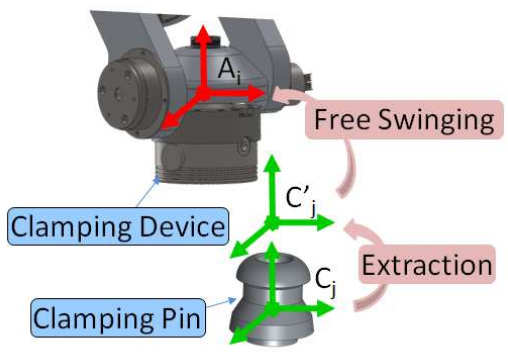

Fig. 7: Extraction process

BE link as the end effector can be established numerically [10]. A whole swinging movement has three processes: the extraction of the clamping device, the free swinging of the limb and the insertion of the clamping device.

In our case, the trajectory following of the swinging BE link is required only during the extraction and the insertion of clamping devices. A practical approach is used for realizing such small range motions of the concerned BE links during these processes.

Fig. 7 shows an example of extraction processes. The frame $\mathscr{R}_{C_{j}}(j=1,2, \ldots)$ is located on the clamping pin. The pose of the frame is described by $x_{c_{j}}\left(x_{C_{j}} ; y_{C_{j}} ; z_{C_{j}} ; \alpha_{C_{j}}\right.$; $\left.\beta_{C_{j}} ; \gamma_{C_{j}}\right)_{(j=1,2, \ldots)}$ where $j$ is the index of the clamping pin. For every clamping pin, there is also a frame $\mathscr{R}_{C^{\prime}}$ $(j=1,2, \ldots)$ described by $x_{c_{j}}^{\prime}$ which corresponds the pose that the clamping device can begin to move freely.

The extraction movement is a small range movement which brings the limb frame $\mathscr{R}_{A_{i}}$ from the frame $\mathscr{R}_{C_{j}}$ to the frame $\mathscr{R}_{C_{j}^{\prime}}$ ( $i$ is the index of the swinging limb, $j$ is the index of the released pin). By keeping the actuator variables $q L_{L_{i}}$ and $q R_{L_{i}}$ to be constants, this limb and the PP all together can be considered as a rigid body. Thus the required extraction movement of the concerned BE link can be obtained by controlling the trajectory of the PP.

Instead of moving the PP from the detaching pose $x_{1}$ to the attaching pose $x_{2}$ directly, two intermedium poses $x_{1}^{\prime}$ and $x_{2}^{\prime}$ are introduced to the trajectory of the PP.

From the pose $x_{1}$ to the pose $x_{1}^{\prime}$, the movement of the PP is generated by actuators located in the three supporting limbs. Meanwhile the actuators in the swinging limb remain constants $\left[q L_{L_{i}}^{\alpha}, q R_{L_{i}}^{\alpha}\right]^{T}$. So that the BE link of this limb follows a shifted trajectory of the PP in order to move from the pose $x_{c_{j}}$ to the pose $x_{c_{j}}^{\prime}$.

During the free swinging of the limb, the PP moves from the pose $x_{1}^{\prime}$ to the pose $x_{2}^{\prime}$. And the actuators of the swinging limb vary from $\left[q L_{L_{i}}^{\alpha}, q R_{L_{i}}^{\alpha}\right]^{T}$ to $\left[q L_{L_{i}}^{\beta}, q R_{L_{i}}^{\beta}\right]^{T}$.

At last, for inserting the clamping device, a reversed process of the extraction is applied.

\section{ILLUSTRATIVE EXAMPLES}

\section{A. Step Between Two Irregular Supporting Patterns}

In this example, the two supporting patterns are not arranged in a regular way: the clamping pins are not in one plane, and they are oriented to different direction. On

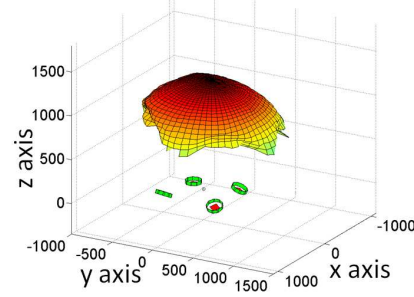

(a) With supporting pattern $\alpha$

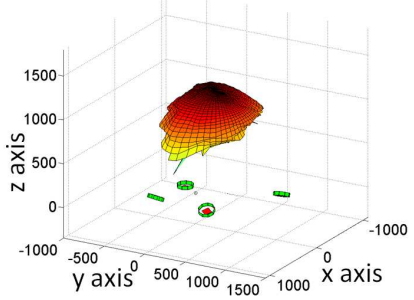

(b) With supporting pattern $\beta$
Fig. 8: Estimated reachable workspace with irregular pattern (dimensions of the axes in all figures are in millimeters)

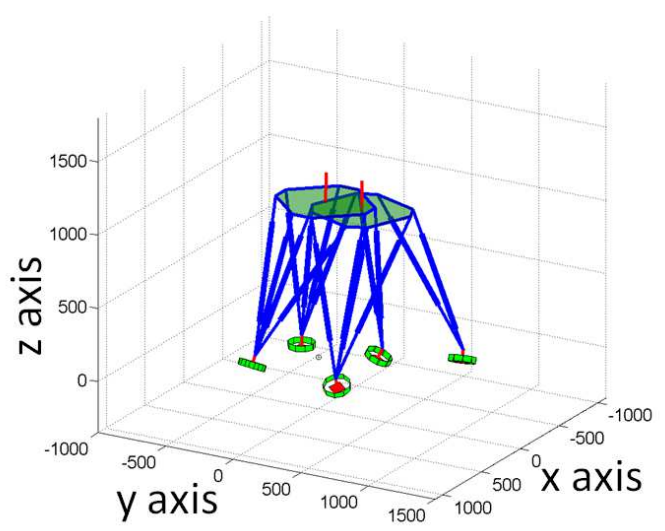

Fig. 9: Detaching and attaching configurations

account of the constrains formulated by (6) and (7), the estimated reachable workspaces of the PKMs under the two supporting patterns are illustrated in Fig. 8. The boundary of the illustrated reachable workspace is determined by using a numerical spherical search method [12].

The found corresponding detaching and attaching configurations are shown in Fig. 9. In this test, REMORA shows the capacity to work on the discontinued surface with moderate curvature, which means the robot is able to adapt to the form of its supporting media.

\section{B. Sequence of Steps under Extra Constrains}

In this example, REMORA takes a sequence of steps in order to work at the next operating position. The locomotion gait of the test is shown in Fig. 10. Extra contrains are applied in the workspaces during the search of key poses of the PP.

In Fig. 11(a), only the initial and the final poses of the robot are illustrated. The blue zone represents the operational workspaces of the tool which are the shifted workspaces of the center point of the PP. The robot should work in the blue zone for achieving the manufacturing operations, however it

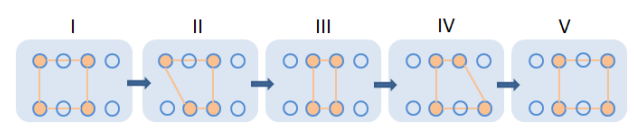

Fig. 10: Locomotion gait 


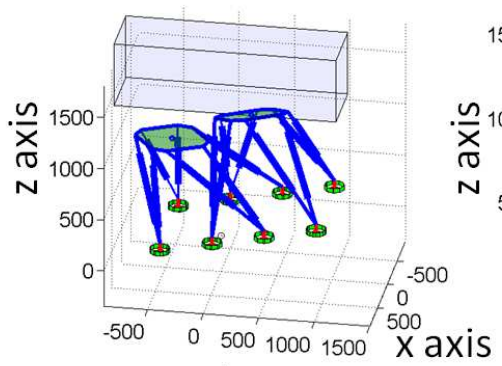

y axis

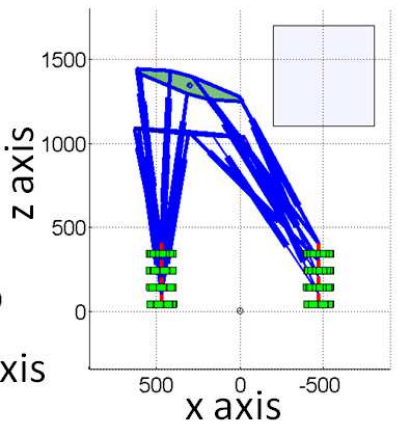

(a) Configurations for initial detachment and final attachment

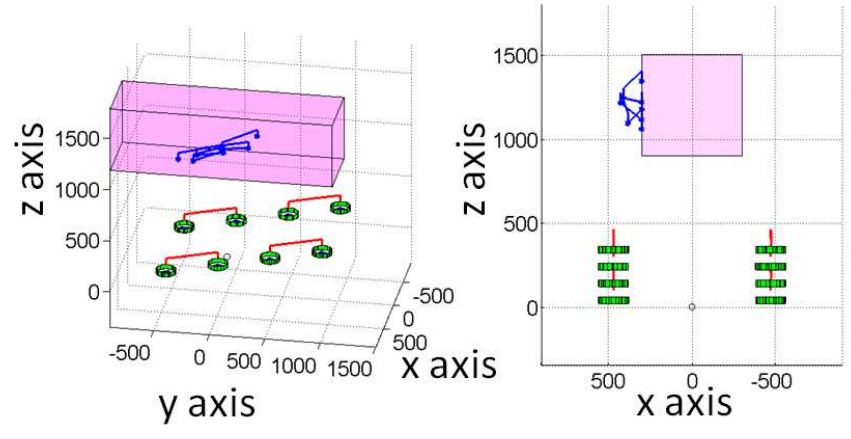

(b) Trajectories of center point of the PP and swinging BE link

Fig. 11: Gaits for changing location of workspace

should not enter this zone during the locomotion movement in order to avoid collisions with the workpieces. The robot is always aside of the blue zone during the locomotion movement.

The pink zone in Fig. 11(b) represents directly the forbidden zone of the center point of the PP. The trajectory of the PP is plotted in blue. The red color is for the trajectories of the BE links. Notice that, with the used objective function, the robot has the tendence to find the key poses in the 'middle' of its workspace. Thanks to the applied constrains, all found poses are outside the pink zone.

The found key poses of the PP is connected by straight line trajectories. The small vertical sections of the PP trajectories are for inserting and exacting the clamping devices. It is interesting to notice that by synchronizing the movement of the PP and the two actuators in the swinging limb, the resulted free swinging trajectories of the concerned BE links are also nearly in the form of straight line.

\section{DISCUSSION AND CONCLUSION}

The preset clamping pins on the supporting media have multiple functions. (1) They offer solid connexion between REMORA and the supporting media, which is essential for high stiffness required operations. (2) The stiffness and accuracy at each operation location can be extended to very large workspaces without building huge guiding structures with high globale precision and rigidity. (3) The clamping pins provide the robot reasonable 'clambering' capacity so that its usage is not limited on the flat floor. (4) By changing forms of the supporting patterns, the robot can realized self-reconfiguration in order to obtain optimized workspaces according to different applications. (5) The clamping pins also avoid other problems such as the localization problem, mapping problem, balancing problem, etc.

Mobile and flexible manufacturing systems are demanded in many industries. In this paper, we present the locomotion approach of a novel quadruped robot called REMORA. This robot is able to carry various effectors for achieving manufacturing tasks in large workspaces. Equipped with lockers on some of the passive joints and clamping devices at the end of its limbs, this quadruped uses eight actuators for achieving manufacturing tasks as well as locomotion tasks. The proposed robot and its working modes are presented. The locking strategy of the robot is formulated as an optimization problem. Also a practical method for managing the limb swinging movement is addressed. At last, the presented locomotion approach is applied on two examples. The tests show that REMORA has a reasonable locomotion capacity for changing the location of its workspaces in workshops. Possessing low degree of kinematic redundancy, the robot can achieve locomotion with respect to some extra constrains in the workspaces. In the future, further studies on the arrangement of supporting pins as well as advanced control methods will be addressed in order to obtain optimized performances for various applications.

\section{REFERENCES}

[1] C. Stillstrom and M. Jackson, "The concept of mobile manufacturing," Journal of Manufacturing Systems, vol. 26, pp. 188-193, 2007.

[2] Y. Yamamoto and X. Yun, "Coordinating locomotion and manipulation of a mobile manipulator," IEEE Transactions on Automatic Control, vol. 39, pp. 1326-1332, 1994.

[3] W.-S. Yoo, J.-D. Kim, and S.-J. Na, "A study on a mobile plaftformmanipulator welding system for horizontal fillet joints," Mechatronics, vol. 2, pp. 835-868, 2001.

[4] H. Wu, H. Handroos, P. Pessi, and J. Hopia, "A mobile hybrid parallel robot with redundant kinematic structure," in Proceedings of the IEEE International Conference on Robotics and Biomimetics, 2006.

[5] J. L. Olazagoitia and S. Wyatt, "New pkm tricept 19000 and its application to flexible manufacturing at aerospace industry," $S A E$ International, vol. 01-3820, p. 2142, 2007.

[6] S. A. Moosavian, A. Pourreza, and K. Alipour, "Kinematics and dynamics of a hybrid serial-parallel mobile robot," in Proceedings of the IEEE International Conference on Robotics and Automation, 2009.

[7] P. GonzalezDeSantos, E. Gracia, and J. Estremera, Walking robots. Springer London, 2007, ch. Quadrupedal Locomotion, pp. 3-32.

[8] K. Yoneda and Y. Ota, "Non-bio-mimetic walkers," The International Journal of Robotics Research, vol. 22, No. 34, pp. 241-249, 2003.

[9] J. M.Sabater, R. J. Saltaren, R. Aracil, E. Yime, and J. M. Azorin, "Teleoperated parallel climbing robots in nuclear installations," Industrial Robot: An International Journal, vol. 33, pp. 381-386, 2006.

[10] H. Yang, S. Krut, C. Baradat, and F. Pierrot, "On the design of mobile parallel robots for large workspace applications," in Proceedings of the ASME International Design Engineering Technical Conferences, 2011.

[11] D. Stewart, "A platform with six degrees of freedom," Aircraft Engineering and Aerospace Technology, vol. 38 Issue:4, pp. 30-35, 1966.

[12] I. A. Bonev and J. Ryu, "A new approach to orientation workspace analysis of 6-dof parallel manipulators," Mechanism and Machine Theory, vol. 36, pp. 15-28, 2001. 